\title{
Control of spontaneous spiral formation in a zwitterionic micellar medium $\uparrow$
}

\author{
Federico Rossi, ${ }^{* a}$ Rosario Varsalona, ${ }^{b}$ Nadia Marchettini ${ }^{c}$ and Maria Liria Turco Liveri ${ }^{* d}$ \\ Received 12th March 2011, Accepted 7th July 2011 \\ DOI: $10.1039 / \mathrm{c} 1 \mathrm{sm} 05439 \mathrm{~b}$
}

\begin{abstract}
The transition from planar fronts, trigger waves or solitary pulses to spirals in excitable media, has attracted increasing interest in the past few decades, mainly because of its relevance for biological and medical applications. In this paper we describe a new and convenient method for spiral generation starting from symmetric wavefronts. By using the micelle-forming zwitterionic surfactant $N$-tetradecyl$N, N$-dimethylamine oxide in a Belousov-Zhabotinsky solution, it is possible to control to a large extent the domains where spirals can be spontaneously generated. The mechanism responsible for the wavefront break up lies in the interaction of the propagating waves with the unexcitable regions formed by the interaction of the surfactant with some of the Belousov-Zhabotinsky key intermediates.
\end{abstract}

\section{Introduction}

Spiral formation and propagation in excitable media is a widespread phenomenon in several biological systems. Simple chemical reactions, such as the well known Belousov-Zhabotinsky (BZ), ${ }^{1,2}$ are often used to study the basic mechanisms responsible for spiral generation in homogeneous or heterogeneous media. In fact, thanks to its characteristics, the BZ reaction, when coupled with diffusive processes, can easily generate oxidation waves in either open or batch reactors; in turn, these waves can break to produce clockwise and counterclockwise rotating spirals. This process has been invoked as a cause of spatio-temporal chaos in spatially extended excitable media, and understanding its intimate mechanisms could shed light on a series of problems with relevance for biology, medicine and physiology. ${ }^{3-7}$

Spontaneous spiral formation was observed in homogeneous $\mathrm{BZ}$ reactions where waves propagate into the vulnerable region of preceding pulses ${ }^{8,9}$ and simulations showed that spirals can be obtained from the combination of the diffusion of reactants with oscillatory kinetics in open reactors with immobilized catalysts. ${ }^{10}$ Generally, the most employed method to break up symmetric fronts is to apply a mechanical stimulus or impose a physical obstacle to a propagating wave or pulse, ${ }^{11-13}$ nevertheless, in recent years there was a certain interest in finding more elegant and less invasive methods. Ruisi and $\mathrm{Wang}^{14}$ proposed a new experimental system where the coupling of two BZ-type oscillators, with different organic substrates, could generate scattered

aDipartimento di Chimica, Università di Siena, Siena. E-mail: f.rossi@ unisi.it

${ }^{b}$ Dipartimento di Chimica, Università di Palermo, Palermo

${ }^{c}$ Dipartimento di Chimica, Università di Siena, Siena

${ }^{d}$ Dipartimento di Chimica, Università di Palermo, Palermo. E-mail. tliveri@unipa.it

$\dagger$ Electronic supplementary information (ESI) available. See DOI: $10.1039 / \mathrm{c} 1 \mathrm{sm} 05439 \mathrm{~b}$ areas of low excitability, which in turn create free ends in planar waves. Toth et al. ${ }^{15}$ used the photosensitive properties of a ruthenium catalyzed $\mathrm{BZ}$ reaction to create spirals by over imposing a mask with a network of areas at different light intensity. Finally, Wang et al., ${ }^{16}$ starting from the results of Maselko et al., ${ }^{17,18}$ found that the number of resin beads can be a bifurcation parameter for spiral generation in a discrete medium (BZ + cation exchange resins). In this paper we present a new system where the coupling of the $\mathrm{BZ}$ reaction with the zwitterionic surfactant $N$-tetradecyl- $N, N$-dimethylamine oxide $\left(\mathrm{C}_{14} \mathrm{DMAO}\right)$ can generate spirals in the medium simply by tuning some experimental parameters, such as the reactants' concentration or the number of micelles in solution. This system presents a few advantages with respect to the aforementioned examples: $i$ ) simple chemistry, being only one oscillator present; ii) homogeneous reaction environment at the macroscale; iii) no external actions are required to break fronts.

The present work follows previous studies on the combination of chemical oscillators with surfactant molecules able to selfassembly in various aggregates, such as micelles, ${ }^{19-26}$ bilayers ${ }^{27,28}$ or polymeric clusters. ${ }^{29,30}$ In particular, a few years ago, we found that a $\mathrm{C}_{14} \mathrm{DMAO}$ micellar system is able to induce an induction period (IP) in a ferroin catalyzed BZ oscillator, when the surfactant concentration overcomes a certain threshold, $\left[\mathrm{C}_{14} \mathrm{DMAO}\right]_{\mathrm{cr}}$; the value of the latter depending on the experimental conditions. ${ }^{31}$ Here we present results about fronts and waves propagation in pseudo bi-dimensional spatially extended reactors, where, in appropriate conditions, the new features of the $\mathrm{BZ} / \mathrm{C}_{14} \mathrm{DMAO}$ system are responsible for spiral generation.

\section{Results}

In our previous work, ${ }^{31}$ we investigated the influence of $\mathrm{C}_{14} \mathrm{DMAO}$ on the temporal oscillation of a well-stirred batch BZ reaction, over a wide surfactant concentration. The oscillator showed a threshold behavior with respect to $\left[\mathrm{C}_{14} \mathrm{DMAO}\right]$, and in 
our experimental conditions $\left([\mathrm{MA}]=0.03 \mathrm{~mol} \mathrm{dm}^{-3},\left[\mathrm{NaBrO}_{3}\right]\right.$ $=0.12 \mathrm{~mol} \mathrm{dm}^{-3}$, [ferroin $]=5.0 \times 10^{-4} \mathrm{~mol} \mathrm{dm}^{-3},\left[\mathrm{H}_{2} \mathrm{SO}_{4}\right]=$ $0.30 \mathrm{~mol} \mathrm{dm}^{-3}$ ) an induction period appeared beyond a surfactant critical concentration $\left[\mathrm{C}_{14} \mathrm{DMAO}\right]_{\mathrm{cr}}=5 \times 10^{-3} \mathrm{~mol} \mathrm{dm}^{-3}$. Fig. 1 shows the typical dynamics of a well stirred ferroin catalyzed BZ reaction before (a) and after (b) the appearance of the IP. During the induction period, the catalyst was found to be in the oxidized form (ferriin, $\mathrm{Fe}(o \text {-phen })_{3}^{3+}$ ), meaning that there was not enough 3-brominated organic substrate to reduce it. The induction period sensibly decreased and eventually disappeared, upon the addition of bromide ions with the subsequent generation of $\mathrm{Br}_{2}$, which could brominate malonic acid, according to the reaction scheme

$$
\begin{aligned}
& \mathrm{BrO}_{3}^{-}+5 \mathrm{Br}^{-}+6 \mathrm{H}^{+} \rightarrow 3 \mathrm{Br}_{2}+3 \mathrm{H}_{2} \mathrm{O} \\
& \mathrm{CH}_{2}(\mathrm{COOH})_{2} \rightleftharpoons(\mathrm{COOH}) \mathrm{CHC}(\mathrm{OH})_{2}
\end{aligned}
$$

$$
(\mathrm{COOH}) \mathrm{CHC}(\mathrm{OH})_{2}+\mathrm{Br}_{2} \rightarrow \mathrm{BrCH}(\mathrm{COOH})_{2}+\mathrm{H}^{+}+\mathrm{Br}^{-}
$$

Experimental results, together with kinetic simulations, led us to consider the interaction of the bromine with the surfactant (both in its monomeric and micellized forms), as the key process responsible for the appearance of the IP. We will discuss the role of $\mathrm{Br}_{2}$ in more details in the section 3.1.

In the experiments in pseudo-bidimensional reactors, $\left[\mathrm{C}_{14} \mathrm{DMAO}\right]_{\mathrm{cr}}$ was found to be smaller by a factor of two, about 2 $\times 10^{-3} \mathrm{~mol} \mathrm{dm}^{-3}$, probably because of the effects of the glass windows on the reaction dynamics and because of the absence of air on top of the reactive solution. The spatial analogue corresponding to the behavior reported in Fig. 1 is depicted in Fig. 2. Panels A-C show the spatio-temporal evolution of a $\mathrm{BZ}$ reaction in the absence of the micelles, regular oxidation waves travel across a reduced (red) medium starting from the edges of the reactor. Panels D-F are snapshots of the dynamic evolution of a $\mathrm{BZ}$ reaction when the concentration of the surfactant overcomes $\left[\mathrm{C}_{14} \mathrm{DMAO}\right]_{\mathrm{cr}}$, in this case the reaction medium appears in an oxidized (blue) status for an interval comparable with the

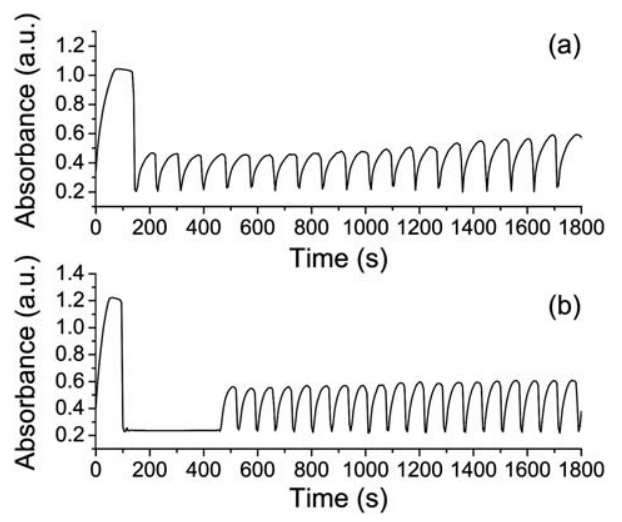

Fig. 1 The effect of the surfactant $\mathrm{C}_{14} \mathrm{DMAO}$ on a well stirred batch ferroin catalyzed $\mathrm{BZ}$ reaction: an oxidized induction period appears above a critical concentration of surfactant. (a) $\left[\mathrm{C}_{14} \mathrm{DAMO}\right]=0 \mathrm{~mol}$ $\mathrm{dm}^{-3}$; (b) $\left[\mathrm{C}_{14} \mathrm{DAMO}\right]=5 \times 10^{-3} \mathrm{~mol} \mathrm{dm}^{-3}$. For both systems: $[\mathrm{MA}]=$ $0.03 \mathrm{~mol} \mathrm{dm}^{-3},\left[\mathrm{NaBrO}_{3}\right]=0.12 \mathrm{~mol} \mathrm{dm}^{-3}$, [ferroin] $=5.0 \times 10^{-4} \mathrm{~mol}$ $\mathrm{dm}^{-3},\left[\mathrm{H}_{2} \mathrm{SO}_{4}\right]=0.30 \mathrm{~mol} \mathrm{dm}{ }^{-3}$. length of the induction period in stirred reactors. When the system shifts towards the oscillatory state, a reductive front (RF) travels from the edges of the reactor towards the center (white arrows in Fig. 2E) and restores the medium to the usual reduced state; oxidative pulses and waves (OW) immediately appear and follow the front (red arrows in Fig. 2E). By using an analogy, the dynamics can be depicted as a pursuit, where the oxidative waves chase the fleeing reductive front.

By varying the concentrations of the chemicals in solution, the relative propagation velocity of the reductive front $\left(v_{\text {red }}\right)$ and oxidation waves $\left(v_{\mathrm{ox}}\right)$ can be controlled to a certain extent, in particular the system can be tuned such that the chaser catches the preceding front and the planar OWs turn into spirals. Fig. 3 illustrates the generation of two counter rotating spirals when $v_{\mathrm{ox}}$ is larger than $v_{\text {red }}$ (the movie is available as supporting material to the present paper $\dagger$ ). In panels $\mathrm{A}-\mathrm{C}$, an oxidative pulse travels in the reduced medium left behind by the reduction front, until it reaches the bright area where the catalyst is oxidized. When the two moving structures get in touch they seem to fuse for a short interval, after which they separate and the reduced area finally disappears. The nature of the RF is still unclear (we will discuss this aspect in more detail in section 3.2) but it is evident that it has an inhibiting effect on the propagation of the OW. In fact, $v_{\mathrm{ox}}$ decreases when the wave approaches the reduced area with a simultaneous flattening of the pattern curvature (a phenomenon that is reminiscent of bunching and merging waves in the BZ/cyclo-hexanedione systems ${ }^{32}$ ). Panels D-F finally show that the disappearance of the oxidized area typical of the IP leaves the oxidation pulse with two free ends, which can curl to form rotating spirals. From this point the reaction dynamics continue as a regular BZ system and spiral patterns persist until the end of the reaction. In most of the cases the interaction between the two fronts generates two coupled spirals, nevertheless when the OW initiates simultaneously (in time) and very close (in space) to the reduction front, a single spiral tends to appear; probably this is dictated by the geometry of the interaction and the angle of contact between the fronts.

Once the composition of the reactive BZ mixture is kept constant, the concentration of the surfactant in solution represents the key parameter that can be tuned to induce spiral generation. Below $\left[\mathrm{C}_{14} \mathrm{DMAO}\right]_{\mathrm{cr}}$ the reductive front is obviously not present and regular waves travel across the reduced medium. When the induction period appears, there exists a [ $\left.\mathrm{C}_{14} \mathrm{DMAO}\right]$ interval where $v_{\text {red }}$ is larger than (or equal to) $v_{\text {ox }}$ and the two fronts never get in touch (the behavior depicted in Fig. 2). However, in a stirred reactor, by augmenting the concentration of surfactant in solution, the length of the induction period increases until a plateau, ${ }^{31}$ this is true also for experiments in pseudo-bidimensional reactors where the longer persistence of the induction period is reflected in a slower RF. The behavior of the system with respect to $\left[\mathrm{C}_{14} \mathrm{DMAO}\right]$ is sketched in Fig. 4 , where the speed of the waves and fronts calculated by means of spacetime plots are reported as a function of the surfactant concentration. Additions of the surfactant below the critical micelles concentration (cmc, $6.3 \times 10^{-5} \mathrm{~mol} \mathrm{dm}^{-3}{ }^{31}$ ) do not significantly influence $v_{\mathrm{ox}}$, while a progressive decrease until a plateau is detected upon further additions; the same is true for $v_{\text {red }}$ except that it does not seem to reach a plateau in the concentration range investigated. In the absence of a catalytic micellar effect, 

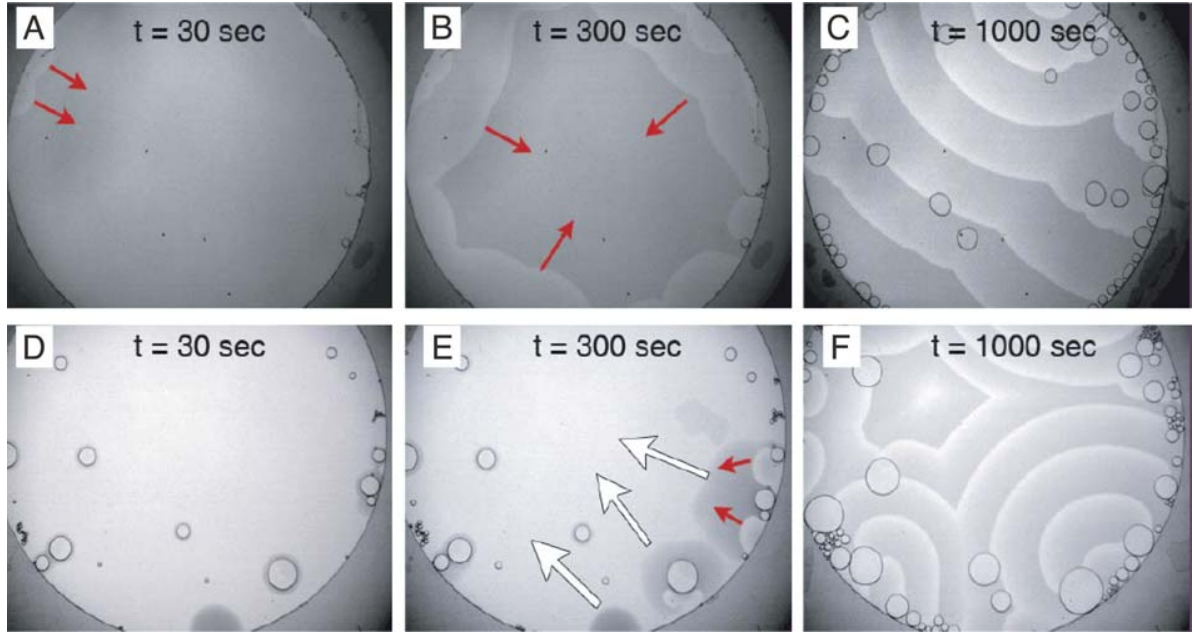

Fig. 2 Spatio-temporal effects of the surfactant $\mathrm{C}_{14} \mathrm{DMAO}$ on an unstirred shallow layer of a batch ferroin catalyzed $\mathrm{BZ}$ reaction. (A)-(C) Oxidation waves travelling in a reduced medium when $\left[\mathrm{C}_{14} \mathrm{DAMO}\right]=0 \mathrm{~mol} \mathrm{dm}^{-3}$; frame size $\left(\mathrm{mm}^{2}\right) 26 \times 22$. (D)-(F) Oxidized induction period and development of a reduction front and oxidation waves when $\left[\mathrm{C}_{14} \mathrm{DAMO}\right]=2 \times 10^{-3} \mathrm{~mol} \mathrm{dm}^{-3}$; frame size $\left(\mathrm{mm}^{2}\right) 24 \times 20$. For both systems: $[\mathrm{MA}]=0.03 \mathrm{~mol} \mathrm{dm}{ }^{-3}$, $\left[\mathrm{NaBrO}_{3}\right]=0.12 \mathrm{~mol} \mathrm{dm}^{-3},[$ ferroin $]=3.0 \times 10^{-3} \mathrm{~mol} \mathrm{dm}^{-3},\left[\mathrm{H}_{2} \mathrm{SO}_{4}\right]=0.30 \mathrm{~mol} \mathrm{dm}^{-3}$.

like in the case of the anionic surfactant $\operatorname{SDS}^{22}$ or $\mathrm{AOT},{ }^{26}$ a general decrease of the speed of waves and fronts is expected due to the increased viscosity of the medium, in section 3.2 we will account for the more pronounced effect observed on the RF. In the interval $2 \times 10^{-3} \mathrm{~mol} \mathrm{dm}^{-3}<\left[\mathrm{C}_{14} \mathrm{DMAO}\right]<3 \times 10^{-3} \mathrm{~mol}$ $\mathrm{dm}^{-3}$, the IP is present but $v_{\text {red }} \geq v_{\text {ox }}$ and spirals do not appear. Finally, when $\left[\mathrm{C}_{14} \mathrm{DMAO}\right]$ is larger than $3 \times 10^{-3} \mathrm{~mol} \mathrm{dm}^{-3}$ spirals are always present, except for one concentration where $v_{\text {ox }}$ drops down to $1.75 \mathrm{~mm} \mathrm{~min}^{-1}$ for unknown reasons.

Other parameters can be tuned in order to trigger spirals in the $\mathrm{BZ}$ medium. For example, it is well established ${ }^{33}$ that the speed of a wave front in reactions where autocatalysis is coupled with diffusion is proportional to the square root of the diffusion coefficient of the autocatalytic species and to the kinetic constant of the rate determinant step of the autocatalytic process; for the $\mathrm{BZ}$ reaction in particular, Field and Noyes ${ }^{34}$ determined that the velocity of the oxidation waves also depends on

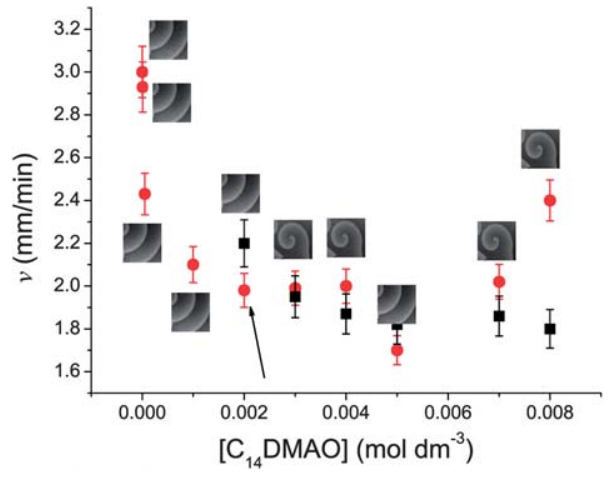

Fig. 4 The behavior of $v_{\mathrm{ox}}(\mathbf{0})$ and $v_{\text {red }}(\mathbf{\square})$ upon the increasing concentration of surfactant in solution. $[\mathrm{MA}]=0.03 \mathrm{~mol} \mathrm{dm}^{-3},\left[\mathrm{NaBrO}_{3}\right]$ $=0.12 \mathrm{~mol} \mathrm{dm}{ }^{-3}$, [ferroin $]=3.0 \times 10^{-3} \mathrm{~mol} \mathrm{dm}^{-3},\left[\mathrm{H}_{2} \mathrm{SO}_{4}\right]=0.30 \mathrm{~mol}$ $\mathrm{dm}^{-3}$. The arrow indicates the appearance of the induction period, small insets sketch the spatio-temporal behavior of the system.
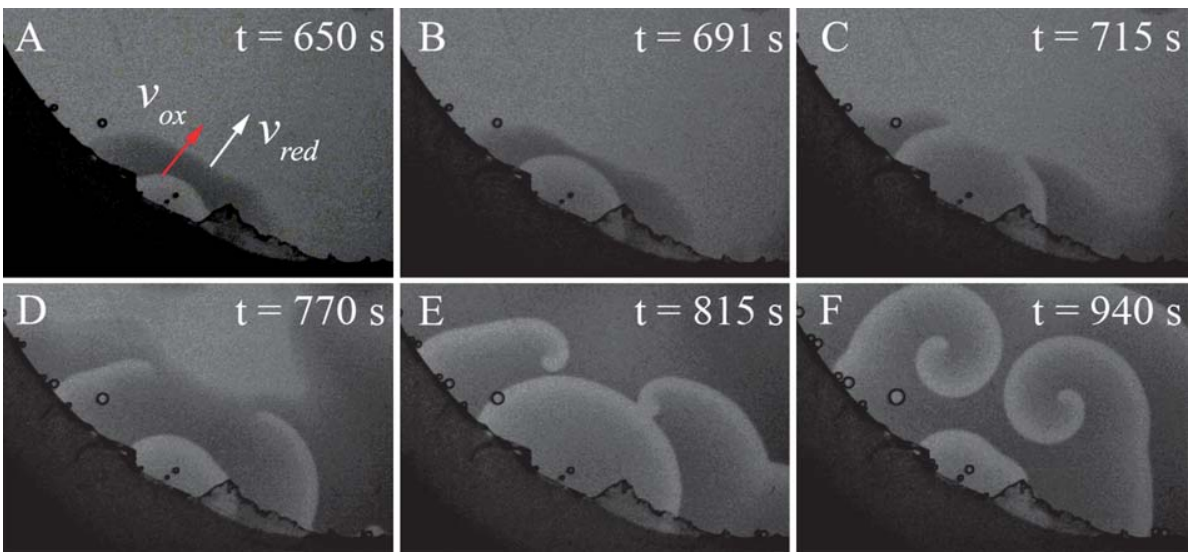

Fig. 3 The process of spontaneous spiral formation when $v_{\mathrm{ox}}>v_{\text {red }}$. The oxidation wave rapidly approaches the reduction front (A), it slows down when entering in the refractory zone close to the front (B), finally the two patterns get in touch and the front annihilates a portion of the wave (C, D) thus generating two counter rotating spirals $(\mathrm{E}, \mathrm{F})$. Frame size $\left(\mathrm{mm}^{2}\right) 7 \times 5 .\left[\mathrm{C}_{14} \mathrm{DMAO}\right]=6 \times 10^{-3} \mathrm{~mol} \mathrm{dm}^{-3},[\mathrm{MA}]=0.03 \mathrm{~mol} \mathrm{dm}{ }^{-3},\left[\mathrm{NaBrO}{ }_{3}\right]=0.12 \mathrm{~mol}$ $\mathrm{dm}^{-3}$, [ferroin] $=3.0 \times 10^{-3} \mathrm{~mol} \mathrm{dm}^{-3},\left[\mathrm{H}_{2} \mathrm{SO}_{4}\right]=0.30 \mathrm{~mol} \mathrm{dm}^{-3}$. The movie of the process is available as supporting material. $\dagger$ 
the concentration of two of the main reagents, that is $v_{\mathrm{ox}}=2\left(k_{\mathrm{a}} D_{\mathrm{a}}\right)^{1 / 2}\left(\left[\mathrm{H}^{+}\right]\left[\mathrm{BrO}_{3}^{-}\right]\right)^{1 / 2}$. Nevertheless, for the sake of simplicity we decided to keep constant the composition of the BZ mixture, i.e. the concentration of the main reagents and we only determined the lower threshold values of sulfuric acid and bromate below which spirals disappeared, namely $\left[\mathrm{H}_{2} \mathrm{SO}_{4}\right]=$ $0.20 \mathrm{~mol} \mathrm{dm}^{-3}$ and $\left[\mathrm{BrO}_{3}^{-}\right]=0.08 \mathrm{~mol} \mathrm{dm}^{-3}$ (with $\left[\mathrm{C}_{14} \mathrm{DMAO}\right]=$ $3 \times 10^{-3} \mathrm{~mol} \mathrm{dm}^{-3}$ and all the other reagents were fixed).

\section{Discussion}

In order to understand the behavior of the $\mathrm{BZ} / \mathrm{C}_{14} \mathrm{DMAO}$ system in spatially extended reactors, we focused our attention on two phenomena particularly relevant for the spiral formation mechanism, namely the interaction of bromine with the surfactant and the development of the reductive front. The two processes are related one to each other and the presence of the reductive front, in particular, is consequent to the formation of the induction period.

\subsection{Interaction of $\mathrm{Br}_{2}$ with $\mathrm{C}_{14} \mathrm{DMAO}$}

Nostictzius and coworkers ${ }^{35}$ recently pointed out that ferriin does not react with malonic acid but it can be reduced by bromomalonic acid (BrMA) to yield $\mathrm{Br}^{-}$and, according to the FKN mechanism, ${ }^{36}$ reset the oscillatory $\mathrm{BZ}$ cycle. As reactions R1-R3 are the main source of BrMA in solution, ${ }^{37,38}$ the role of bromine appears to be crucial in the ferroin catalyzed systems, and its segregation from MA likely appeared to be responsible for the appearance of the oxidized induction period. Moreover, as mentioned in section 2, previous experiments ${ }^{31}$ led us to suspect a strong interaction between $\mathrm{Br}_{2}$ and the micellized surfactant. We therefore postulated the formation of an adduct between the surfactant and the molecular bromine (reaction A1) to account for the inhibition of the bromination reaction of malonic acid and consequently for the appearance of the induction period itself.

$$
\mathrm{C}_{14} \mathrm{DMAO}+\mathrm{Br}_{2} \rightleftharpoons \mathrm{K}-\mathrm{C}_{14} \mathrm{DMAO}-\mathrm{Br}_{2}
$$

From spectrophotometric measurements, it was found that the spectrum of molecular bromine when the surfactant is added to the solution presents a new absorption band with a maximum at $\lambda=271.5 \mathrm{~nm}$. In the presence of $n(\pi)$-donors/acceptors, the appearance of a new band in the UV-Vis region, is generally attributed to the formation of charge-transfer complexes (CTC). Typical CTC absorption bands, for example, have been observed when bromine interacts with a variety of $\pi$-donors, like benzene ${ }^{39}$ or cyclohexene $e^{40,41}$ and $n$-donors like sodium bis(2-ethylexhyl) sulfosuccinate (AOT) ${ }^{42} \mathrm{C}_{14} \mathrm{DMAO}$, like most of the aminooxide surfactants, is a weak base and because of its chemical structure it can be considered an analogue of an aliphatic amine, i.e. an $n$-donor equivalent. In fact, during the reaction of tertiary amines with bromine, the formation of stable CTC complexes has been identified as a crucial step before the fast intramolecular oxidation that leads to the products. ${ }^{43,44}$ Fig. 5 shows the evidence of a CTC formation, where the absorbance at $\lambda=$ $271.5 \mathrm{~nm}$ increases with the concentration of the surfactant, passing from 0.90 a.u. at $\left[\mathrm{C}_{14} \mathrm{DMAO}\right]=1 \times 10^{-3} \mathrm{~mol} \mathrm{dm}^{-3}$ to 1.99 a.u. at $\left[\mathrm{C}_{14} \mathrm{DMAO}\right]=6 \times 10^{-3} \mathrm{~mol} \mathrm{dm}^{-3}$.

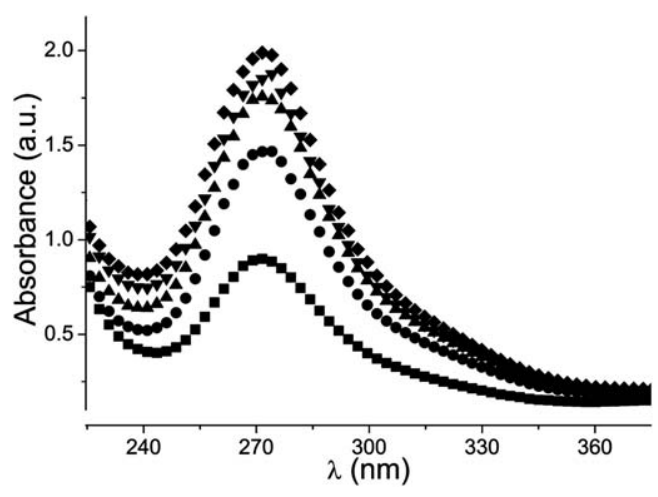

Fig. 5 Typical spectral results showing the formation of a CTC between the bromine and the surfactant; $\left[\mathrm{Br}_{2}\right]=6.0 \times 10^{-4} \mathrm{~mol} \mathrm{dm}{ }^{-3},\left[\mathrm{H}_{2} \mathrm{SO}_{4}\right]=$ $0.3 \mathrm{~mol} \mathrm{dm}^{-3},(\boldsymbol{\square})\left[\mathrm{C}_{14} \mathrm{DMAO}\right]=1 \times 10^{-3} \mathrm{~mol} \mathrm{dm}^{-3},(\bullet)\left[\mathrm{C}_{14} \mathrm{DMAO}\right]=$ $3 \times 10^{-3} \mathrm{~mol} \mathrm{dm}^{-3},(\mathbf{\Delta})\left[\mathrm{C}_{14} \mathrm{DMAO}\right]=4 \times 10^{-3} \mathrm{~mol} \mathrm{dm}^{-3},(\boldsymbol{\nabla})$ $\left[\mathrm{C}_{14} \mathrm{DMAO}\right]=5 \times 10^{-3} \mathrm{~mol} \mathrm{dm}^{-3},(\diamond)\left[\mathrm{C}_{14} \mathrm{DMAO}\right]=6 \times 10^{-3} \mathrm{~mol}$ $\mathrm{dm}^{-3}, t \sim 24{ }^{\circ} \mathrm{C}$.

At the equilibrium, the concentrations of the complex and of the free reactants are unknown, therefore is impossible to calculate the equilibrium constant $(K)$ for the reaction A1 by means of the classic mass action law and a procedure derived from ref. 42 and 45 and adapted to our purpose was used. The detailed description of the method can be found in the supporting material of the present paper. $\dagger$ By applying this procedure we found that $K=(5.75 \pm 0.36) \times 10^{2} \mathrm{~mol}^{-1} \mathrm{dm}^{3}$ and that the molar extinction coefficient of the complex $\varepsilon=(4.16 \pm 0.03)$ $\times 10^{3} \mathrm{~cm}^{-1} \mathrm{~mol}^{-1} \mathrm{dm}^{3}$. It has to be noted that the values for $K$ and $\varepsilon$ are an estimation, which accounts for both the monomeric and the micellar form of the surfactant, with spectrophotometric data suggesting a stronger interaction with the monomers rather than with the micelles (see the supporting material for details $\dagger$ ).

The value of the complex formation constant is lower than that used for simulations in our previous work (roughly equal to 1000 ), but still it is a confirmation of the role of the surfactant in sequestrating the bromine from the aqueous phase. Moreover the method we used inserts a degree of uncertainty due to the fact that we cannot measure the formation constants for $\mathrm{Br}_{2}$ with $\mathrm{C}_{14} \mathrm{DMAO}$ monomers below the $\mathrm{cmc}$, and the value we found may be re-evaluated to a bigger estimation. In our experiments, we cannot exclude that the surfactant undergoes an oxidation, like in the case of tertiary aliphatic-amines, however, by monitoring the absorbance at $\lambda=271.5 \mathrm{~nm}$ over time, we could not detect any significant decrease in the maximum for prolonged intervals.

In order to further check the role of bromine in the generation of the oxidized induction period, we added an increasing amount of acetone (Ac) to the reactive BZ mixture. Acetone is well known as a bromine scavenger and it has been successfully employed as a feedback regulator in controlling $\left[\mathrm{Br}_{2}\right]$ in some variants of BHC (Bromine Hydrolysis Controlled) oscillators. ${ }^{46}$ The influence of acetone on the length of the IP of a cerium catalyzed $\mathrm{BZ}$ reaction has also been investigated ${ }^{47}$ and it was found that removal of bromine increases the length of the IP and of the number of total oscillations, leaving the frequency and the amplitude almost unaltered. This behavior has been attributed to the fact that acetone and bromoacetone do not react with any 
other species present in solution, ${ }^{48}$ therefore the net result is only a delay in the production of BrMA. In our experimental conditions the adding of an increasing amount of acetone was found to alter all the oscillatory parameters, without inducing the appearance of an IP similar to that obtained with $\mathrm{C}_{14} \mathrm{DMAO}$. The spectrophotometric time series showing the absorbance of the ferroin for different $[\mathrm{Ac}]$ are reported in the supporting material. $\uparrow$ Our findings suggest that in the ferroin catalyzed BZ systems, Ac and/or BrAc interact with other reaction steps of the oscillatory system, thus making the comparison with the surfactant difficult. Anyway, the time series recorded unambiguously show that removal of bromine pushes the global oxidation state of the system towards the oxidized state, with a net prevalence of ferriin.

\subsection{The reduction front}

The existence of reduction waves (RW) has been previously observed for ferroin catalyzed $\mathrm{BZ}$ systems when the ratio [MA]/ $\left[\mathrm{BrO}_{3}^{-}\right]$was relatively low. ${ }^{49-53}$ Smoes $^{49}$ was the first to observe the propagation of reduction waves in such a highly oxidized medium, they originated from point defects and were rapidly followed by faster oxidation waves developing in the reduced medium. RW were considered trigger waves and their origin was connected with a local increase of the $\mathrm{Br}^{-}$concentration, which excites the neighboring points and induces a front of catalyst reduction (red spot), which starts to propagate into the oxidized medium; the velocity of the reduction waves is determined by the rate of $\mathrm{Br}^{-}$generation (see eqn (FKN-C) later in the text) and it was found to increase with [MA] while it was independent from $\left[\mathrm{H}_{2} \mathrm{SO}_{4}\right]$ and $\left[\mathrm{BrO}_{3}^{-}\right]^{49,51}$

Several experiments proved that the reduction front observed in our system has the same nature as the waves observed in the highly oxidized media, except for the fact that the depression of the organic substrate concentration is temporary and the reduction front appears only once following the oxidized induction period. In fact, as previously stated, the presence of the surfactant causes a delay in the bromination of MA for an interval, which depends on [ $\left.\mathrm{C}_{14} \mathrm{DMAO}\right]$; the addition of bromide ions reduces the IP and also influences $v_{\text {red }}$, while $v_{\mathrm{ox}}$ is not significantly influenced. Fig. 6 shows that by increasing the

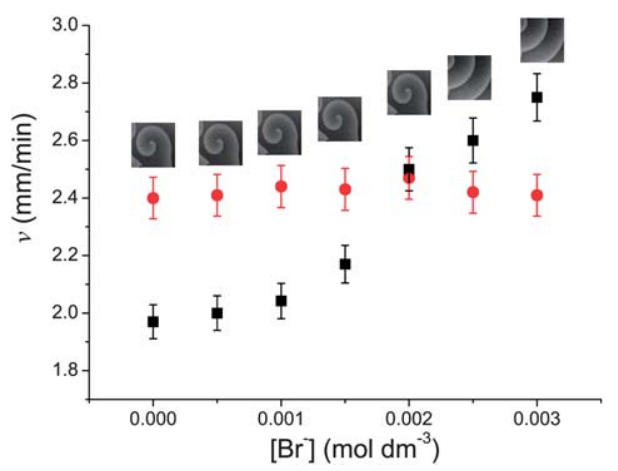

Fig. 6 The behavior of $v_{\mathrm{ox}}(-)$ and $v_{\text {red }}(\square)$ upon the increasing concentration of bromide ions in solution. $\left[\mathrm{C}_{14} \mathrm{DMAO}\right]=8 \times 10^{-3} \mathrm{~mol}$ $\mathrm{dm}^{-3},[\mathrm{MA}]=0.03 \mathrm{~mol} \mathrm{dm}^{-3},\left[\mathrm{NaBrO}_{3}\right]=0.12 \mathrm{~mol} \mathrm{dm}^{-3},[$ ferroin] $=3.0$ $\times 10^{-3} \mathrm{~mol} \mathrm{dm}^{-3},\left[\mathrm{H}_{2} \mathrm{SO}_{4}\right]=0.30 \mathrm{~mol} \mathrm{dm}^{-3}$. The small insets sketch the spatio-temporal behavior of the system.

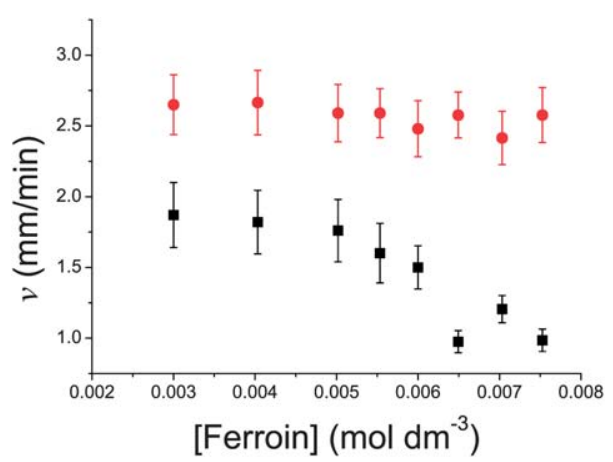

Fig. 7 Behavior of $v_{\text {ox }}(\bullet)$ and $v_{\text {red }}(\boldsymbol{\square})$ upon the increasing concentration of the catalyst in solution. $\left[\mathrm{C}_{14} \mathrm{DMAO}\right]=6 \times 10^{-3} \mathrm{~mol} \mathrm{dm}^{-3}$, $[\mathrm{MA}]=0.03 \mathrm{~mol} \mathrm{dm}^{-3},\left[\mathrm{NaBrO}_{3}\right]=0.12 \mathrm{~mol} \mathrm{dm}^{-3},\left[\mathrm{H}_{2} \mathrm{SO}_{4}\right]=0.30 \mathrm{~mol}$ $\mathrm{dm}^{-3}$.

concentration of bromide at a fixed solution composition $\left(\left[\mathrm{C}_{14} \mathrm{DMAO}\right]=8 \times 10^{-3} \mathrm{~mol} \mathrm{dm}^{-3}\right)$, the velocity of the $\mathrm{RF}$ grows until $\left[\mathrm{Br}^{-}\right]$is about $3 \times 10^{-3} \mathrm{~mol} \mathrm{dm}^{-3}$ when the induction period disappears, moreover at $\left[\mathrm{Br}^{-}\right] \simeq 2.5 \times 10^{-3} \mathrm{~mol} \mathrm{dm}^{-3} v_{\text {red }}$ is larger enough than $v_{\text {ox }}$ to stop the process of spiral formation.

The reduction reaction of the ferriin by the organic substrate represents the process $\mathrm{C}$ in the FKN model ${ }^{36}$ which is responsible for the regeneration of the catalyst and causes an increase of the $\left[\mathrm{Br}^{-}\right]$in solution. This process is represented by the reaction FKN-C

$2 \mathrm{Fe}(\text { phen })_{3}^{3+}+\mathrm{BrMA} \rightarrow f \mathrm{Br}^{-}+2 \mathrm{Fe}(\text { phen })_{3}^{2+}+$ other products (FKN-C)

therefore we also investigated the dependence of $v_{\text {red }}$ upon the concentration of the catalyst in solution. Perusal of Fig. 7 reveals that the velocity of the oxidation waves, as expected, is not influenced by the concentration of the catalyst in solution; on the contrary, $v_{\text {red }}$ sensibly decreases when the reduced form of the catalyst increases. Interestingly, for low values of [ferroin] $(<4 \times$ $10^{-4} \mathrm{~mol} \mathrm{dm}^{-3}$ ) the induction period could not be spectrophotometrically detected in stirred systems, a behavior that reminds us of the threshold like response of the $\mathrm{BZ} / \mathrm{C}_{14} \mathrm{DMAO}$ system to the concentration of the surfactant and of the bromides. ${ }^{31}$

In the light of the mechanism of RF propagation, it can be inferred how the concentration of the surfactant in solution becomes crucial in controlling the propagation velocity of the reducing fronts; when $\left[\mathrm{C}_{14} \mathrm{DMAO}\right]$ increases, the amount of BrMA available for the production of $\mathrm{Br}^{-}$decreases and, since MA is less important for process $\mathrm{C}^{54} v_{\text {red }}$ tends to diminish as shown in Fig. 4. The proposed mechanism also accounts for the anomalous dispersion relation shown by the oxidation waves when approaching the RW. In fact the reducing front leaves behind a high $\left[\mathrm{Br}^{-}\right]$, which is a strong inhibitor for the autocatalytic process responsible for the OW propagation. This is also the reason for the annihilation of the portion of the OW that touches the RW.

\section{Conclusion}

In this paper we introduced a novel way to exploit the combination of self assembling surfactants with the 
Belousov-Zhabotinsky reaction. In our previous works we found that the zwitterionic $\mathrm{C}_{14}$ DMAO prevents the bromination of the organic substrate, thus generating an unusual oxidized induction period in the ferroin catalyzed BZ system. This property has been further investigated and used to induce the spontaneous formation of spirals in a shallow layer of solution.

The mechanism of spiral generation was found to be quite simple and controllable to a large extent, so that by tuning a few parameters of the system it was possible to break symmetric wavefronts and get counter rotating (or in some cases simple) spirals without any mechanical or external actions. The mechanism can be sketched with a few simple steps, namely i) the zwitterionic surfactant sequestrates bromine through the formation of a CTC adduct as described in section 3.1 ; ii) the ferriin cannot be reduced until the BrMA reaches a critical concentration in solution, consequently an oxidized induction period appears; iii) the IP disappears through the propagation of a reductive front (section 3.2) that resets the medium to a reduced form where oxidation waves can propagate; iv) finally, when the parameters are set so that $v_{\mathrm{ox}}$ is larger than $v_{\text {red }}$, the oxidation waves reach the preceding reductive front and generate spirals.

Our method is, in principle, valid for any halogen-based oscillator where the added surfactant can selectively interact with one of the key intermediates. The behavior of iodine, for example, is similar to that of bromine with respect to its ability of forming CTC with amines ${ }^{44}$ and anionic surfactants. ${ }^{42}$ Moreover, the investigation can also be extended to other self-assembling molecules, like the more biologically relevant phospholipid compounds, in order to find behaviors similar to $\mathrm{C}_{14} \mathrm{DMAO}$ by acting on intermediates with different chemical properties (ionic species, radicals, etc.).

\section{Experimental}

Malonic acid (MA), $\mathrm{NaBrO}_{3}$, ferroin (Fe), $\mathrm{NaBr}$, sulfuric acid and bromine were commercial grade reactants (Fluka) and used without further purification. $\mathrm{C}_{14} \mathrm{DMAO}$ was a gift of Prof. $\mathrm{H}$. Hoffmann (Bayreuth Center for Colloids \& Interfaces). Stock solutions were prepared by weight before use. Deionized water from reverse osmosis (Elga, model Option 3), having a resistivity higher than $1 \mathrm{M} \Omega \mathrm{cm}^{-1}$, was used to prepare all solutions. The concentrations of $\mathrm{NaBrO}_{3}\left(0.12 \mathrm{~mol} \mathrm{dm}^{-3}\right), \mathrm{MA}\left(0.03 \mathrm{~mol} \mathrm{dm}{ }^{-3}\right)$ and $\mathrm{H}_{2} \mathrm{SO}_{4}\left(0.30 \mathrm{~mol} \mathrm{dm}{ }^{-3}\right)$ were kept constant for all the experiments while the others were varied in the following ranges: $0<\left[\mathrm{C}_{14} \mathrm{DMAO}\right]<9.0 \times 10^{-2} \mathrm{~mol} \mathrm{dm}^{-3}, 2.8 \times 10^{-4} \mathrm{~mol} \mathrm{dm}^{-3}<$ $[\mathrm{Fe}]<7.0 \times 10^{-3} \mathrm{~mol} \mathrm{dm}^{-3}, 1.0 \times 10^{-3} \mathrm{~mol} \mathrm{dm}^{-3}<[\mathrm{NaBr}]<3.0$ $\times 10^{-3} \mathrm{~mol} \mathrm{dm}^{-3}$.

In order to follow the evolution of spirals and patterns, experiments were performed in a pseudo 2-dimensional reactor. The reactive solution was sandwiched between two $49 \mathrm{~mm}$ diameter boro-silicate optical windows, kept apart by a $100 \mu \mathrm{m}$ thick teflon gasket with an internal diameter of $25 \mathrm{~mm}$. The sample was illuminated from below with a LED light source, while a CCD camera (PiXeLink PL-A774), equipped with a close focus zoom $10 \times$ (Edmund optics) and a band pass interference filter (Edmund optics, $\lambda=510 \mathrm{~nm}$ ), was placed above the sample and connected to a PC for image acquisition. Space-time (ST) plots were built by cutting thin slices (1 pixel) from the same region of every frame of the recorded sequences and pasting them sequentially together in a new image. Every pixel on the time axis represents the image capture sampling time (1 s), and the space axis represents the actual space spanned by the waves. Waves and fronts speeds were calculated from the slope of diagonal lines in ST plots.

Spectrophotometric experiments were performed in quartz cuvettes with a PC-controlled diode-array (Analytic Jena Specord S600) or with a PC-controlled UV-Vis spectrophotometer (Beckman DU 640). Both the instruments were equipped with a magnetic stirring apparatus. Both the absorbance of ferroin $\left(\lambda=510 \mathrm{~nm}, \varepsilon=1.1 \times 10^{4} \mathrm{~mol}^{-1} \mathrm{dm}^{3} \mathrm{~cm}^{-1}\right)$ and ferriin $(\lambda=$ $630 \mathrm{~nm}, \varepsilon=620 \mathrm{~mol}^{-1} \mathrm{dm}^{3} \mathrm{~cm}^{-1}$ ) were collected over time. Experiments on the formation of the CTC complex were performed by following spectrophotometrically the increment in the absorbance of the maximum at $271.5 \mathrm{~nm} v s$. [ $\mathrm{C}_{14} \mathrm{DMAO}$. Every sample was prepared by diluting the proper amount of stock solutions to obtain the desired $\mathrm{C}_{14}$ DMAO concentration $(1.0 \times$ $\left.10^{-3}-6.0 \times 10^{-3} \mathrm{~mol} \mathrm{dm}^{-3}\right)$ and the following initial reactant concentrations: $\left[\mathrm{Br}_{2}\right]=6.0 \times 10^{-4} \mathrm{~mol} \mathrm{dm}{ }^{-3},\left[\mathrm{H}_{2} \mathrm{SO}_{4}\right]=0.3 \mathrm{~mol}$ $\mathrm{dm}^{-3}$.

All experiments were performed at room temperature $\left(\sim 24^{\circ} \mathrm{C}\right)$.

\section{Acknowledgements}

F.R. was supported by a Marie Curie International Outgoing Fellowship within the 7th European Community Framework Programme. The authors thank the Università degli Studi di Palermo for financial support (Research Funds ex quota 60\%). Thanks are also due to Ms. Giulia Gelardi for experimental help and to Mr. Casimiro Caruso for technical support. The gift of $\mathrm{C}_{14}$ DMAO by Prof. H. Hoffmann (University of Bayreuth) is gratefully acknowledged.

\section{References}

1 B. P. Belousov, Sbornik Referatov po Radiatsonno Meditsine, Moscow, 1958, pp. 145-147.

2 A. M. Zhabotinsky, Proc. Acad. Sci. USSR, 1964, 157, 392-395.

3 A. V. Panfilov, Chaos, 1998, 8, 57.

4 A. Winfree, Science, 1994, 266, 1003-1006.

5 S. Alonso, F. Sagues and A. S. Mikhailov, Science, 2003, 299, 1722 1725.

6 T. K. Shajahan, A. R. Nayak and R. Pandit, PLoS One, 2009, 4, e4738.

7 W. Guo, C. Qiao, Z. Zhang, Q. Ouyang and H. Wang, Phys. Rev. E: Stat., Nonlinear, Soft Matter Phys., 2010, 81, 056214-4.

8 M. Gomez-Gesteria, G. Fernandez-Garcia, A. P. Munuzuri, V. PerezMunuzuri, V. I. Krinsky, C. F. Starmer and V. Perez-Villar, Phys. D, 1994, 76, 359-368.

9 J. Dajka, T. Karoly, I. P. Nagy, V. Gaspar and Z. Noszticzius, J. Chem. Soc., Faraday Trans., 1996, 92, 2897-2901.

10 P. Kettunen, T. Amemiya, T. Ohmori and T. Yamaguchi, Phys. Rev. E: Stat. Phys., Plasmas, Fluids, Relat. Interdiscip. Top., 1999, 60, $1512-1515$.

11 A. T. Winfree, Science, 1972, 175, 634-636.

12 J. P. Keener and J. J. Tyson, Phys. D, 1986, 21, 307-324.

13 I. R. Epstein and K. Showalter, J. Phys. Chem., 1996, 100, 1313213147.

14 V. Ruisi and J. Wang, Phys. Rev. E: Stat., Nonlinear, Soft Matter Phys., 2008, 78, 016102-6.

15 R. Toth, B. D. Costello, C. Stone, J. Masere, A. Adamatzky and L. Bull, Phys. Rev. E: Stat., Nonlinear, Soft Matter Phys., 2009, 79, 035101-4.

16 G. Wang, Q. Wang, P. He, S. Pullela, M. Marquez and Z. Cheng, Phys. Rev. E: Stat., Nonlinear, Soft Matter Phys., 2010, 82, 045201. 
17 J. Maselko, J. S. Reckley and K. Showalter, J. Phys. Chem., 1989, 93, 2774-2780.

18 J. Maselko and K. Showalter, Phys. D, 1991, 49, 21-32.

19 V. K. Vanag and I. R. Epstein, Phys. Rev. Lett., 2001, 87, 228301-4.

20 A. Paul, J. Phys. Chem. B, 2005, 109, 9639-9644.

21 L. Sciascia, R. Lombardo and M. L. T. Liveri, J. Phys. Chem. B, 2007, 111, 1354-1360.

22 F. Rossi, R. Lombardo, L. Sciascia, C. Sbriziolo and M. L. T. Liveri, J. Phys. Chem. B, 2008, 112, 7244-7250.

23 F. Rossi and M. L. T. Liveri, Ecol. Modell., 2009, 220, 1857-1864.

24 F. Rossi and M. L. T. Liveri, Colloids Surf., A, 2009, 351, 60-64.

25 V. K. Vanag and I. R. Epstein, in Self-Organized Morphology in Nanostructured Materials, ed. K. Al-Shamery and J. Parisi, Berlin, 2008, pp. 89-113.

26 R. A. Jahan, K. Suzuki, H. Mahara, S. Nishimura, T. Iwatsubo, A. Kaminaga, Y. Yamamoto and T. Yamaguchi, Chem. Phys. Lett., 2010, 485, 304-308.

27 A. Magnani, N. Marchettini, S. Ristori, C. Rossi, F. Rossi, M. Rustici, O. Spalla and E. Tiezzi, J. Am. Chem. Soc., 2004, 126, 11406-11407.

28 F. Rossi, S. Ristori, M. Rustici, N. Marchettini and E. Tiezzi, J. Theor. Biol., 2008, 255, 404-412.

29 L. Sciascia, R. Lombardo and M. L. T. Liveri, Chem. Phys. Lett., 2006, 430, 67-70.

30 L. Sciascia, F. Rossi, C. Sbriziolo, M. L. T. Liveri and R. Varsalona, Phys. Chem. Chem. Phys., 2010, 12, 11674-11682.

31 F. Rossi, R. Varsalona and M. L. T. Liveri, Chem. Phys. Lett., 2008, 463, 378-382.

32 G. Bordyugov, N. Fischer, H. Engel, N. Manz and O. Steinbock, Phys. D, 2010, 239, 766-775.

33 R. Luther, R. Arnold, K. Showalter and J. Tyson, J. Chem. Educ., 1987, 64, 740-742.

34 R. J. Field and R. M. Noyes, J. Am. Chem. Soc., 1974, 96, 2001-2006.

35 L. Hegedus, H. Forsterling, L. Onel, M. Wittmann and Z. Noszticzius, J. Phys. Chem. A, 2006, 110, 12839-12844.
36 R. J. Field, E. Koros and R. M. Noyes, J. Am. Chem. Soc., 1972, 94, 8649-8664.

37 P. Sevcik, M. Snircova and J. Hlavacova, React. Kinet. Catal. Lett., 1994, 52, 161-168.

38 F. Rossi, E. Simoncini, N. Marchettini and E. Tiezzi, Chem. Phys. Lett., 2009, 470, 147-150.

39 R. M. Keefer and L. J. Andrews, J. Am. Chem. Soc., 1950, 72, 46774681.

40 J. E. Dubois and F. Garnier, Spectrochim. Acta, Part A, 1967, 23, 2279-2288.

41 G. Bellucci, R. Bianchini and R. Ambrosetti, J. Am. Chem. Soc., 1985, 107, 2464-2471.

42 L. Garcia-Rio, J. Mejuto, R. Ciri, I. Blagoeva, J. R. Leis and M. Ruasse, J. Phys. Chem. B, 1999, 103, 4997-5004.

43 G. Bellucci, G. Berti, R. Bianchini and L. Orsini, Tetrahedron Lett., 1982, 23, 3635-3638.

44 G. Bellucci, R. Bianchini and R. Ambrosetti, J. Chem. Soc., Perkin Trans. 2, 1987, 39-45.

45 J. Anunziata, J. Singh and J. Silber, Can. J. Chem., 1981, 59, 12911296.

46 A. F. Taylor, Prog. React. Kinet. Mech., 2002, 27, 247-325.

47 I. Berenstein, J. Agreda and D. Barragan, J. Phys. Chem. A, 1999, 103, 9780-9782.

48 R. J. Field and P. M. Boyd, J. Phys. Chem., 1985, 89, 3707-3714.

49 M. L. Smoes, Dynamics of synergetic systems, Bielefeld, Fed. Rep. of Germany, 1980, pp. 80-96.

50 A. M. Zhabotinsky, F. Buchholtz, A. B. Kiyatkin and I. R. Epstein, J. Phys. Chem., 1993, 97, 7578-7584.

51 M. Marek, P. Kastanek and S. C. Mueller, J. Phys. Chem., 1994, 98, $7452-7454$.

52 J. Kosek and M. Marek, Phys. Rev. Lett., 1995, 74, 2134.

53 P. Kastanek, J. Kpsek, D. Snita, I. Schreiber and M. Marek, Phys. D, 1995, 84, 79-94.

54 H. D. Foersterling, L. Stuk, A. Barr and W. D. McCormick, J. Phys. Chem., 1993, 97, 2623-2627. 